\title{
Molecular Characterization and Expression Analysis of the Gene Encoding 3-Hydroxyacyl-CoA Dehydrogenase (EGR-03347) from Echinococcus Granulosus and the Evaluation of the Immune Protection of the Definitive Hosts (dogs)
}

Jinwen Xian

Shihezi University

Ning Wang

State Key Laboratory of sheep genetic improvement and healthy production

Pengpeng Zhao

State Key Laboratory of sheep genetic improvement and healthy prodution

Yanyan Zhang

State Key Laboratory of sheep genetic improvement and healthy production

Jimeng Meng

State Key Laboratory of sheep genetic improvement and healthy production

Xun Ma

Shihezi University

Zhengrong Wang ( $\nabla$ wzrtiger@sina.com )

Shihezi University

Xinwen Bo

Healthcare Improvement Scotland

\section{Research}

Keywords: E. granulosus, Cystic echinococcosis, EGR-03347, Immunolocalization, Immunogenicity, Dog vaccine

Posted Date: June 8th, 2021

DOI: https://doi.org/10.21203/rs.3.rs-579772/v1

License: (c) (i) This work is licensed under a Creative Commons Attribution 4.0 International License.

Read Full License 


\section{Abstract}

Background: Cystic echinococcosis, a serious parasitic zoonosis, is caused by tapeworm (Echinococcus granulosus) larvae. The development of an effective vaccine is a promising strategy to control echinococcosis. E. granulosus has a complete tricarboxylic acid cycle pathway, in which 3-hydroxyacylCoA dehydrogenase (EGR-03347) is a key enzyme.

Methods: In the present study, the cDNA encoding EGR-03347 in Echinococcus granulosus (rEGR-03347) was successfully cloned and the molecular and biochemical characterizations carried out. The immunoreactivity of recombinant EGR-03347 (rEGR-03347) was investigated using western blotting. The immunolocalization of EGR-03347 in different life stages of E. granulosus was determined using specific polyclonal antibody, quantitative real-time reverse transcription polymerase chain reaction was used to analyze their transcript levels in PSCs and 28-day strobilated worms stages. In addition, recombinant protein rEGR-03347 was mixed with the adjuvant Quil A for vaccinating dogs, after three vaccine injections, all the dogs were orally challenge-infected with 100000 protoscoleces of E. granulosus. After 28 days of infection, all the dogs were euthanized and necropsied for collecting and counting $E$. granulosus worms, post-infection the antibody and cytokine were measured for the immunogenicity analysis of this protein.

Results: rEGR-03347 is a highly conserved protein, consisting of 308 amino acids. Recombinant EGR03347 could be identifed in the sera of patients with CE and in mouse anti-rEGR-03347 sera.

Immunofluorescence analysis showed that EGR-03347 mainly localized in the tegument of protoscoleces and adults, and their transcript levels were high in the 28-day strobilated worms. Furthermore, enzymelinked immunosorbent assays post-infection showed that IgG gradually increased after the first immunization with rEGR-03347 compared with the control group, rEGR-03347 changed the interferon gamma and interleukin 4 levels. We observed an $87.2 \%$ reduction in E. granulosus numbers and $66.7 \%$ inhibition of the segmental development of E. granulosus in the rEGR-03347-vaccinated dogs compared with the nonvaccinated controls.

Conclusions: This is the first report characterizing a 3-hydroxyacyl-CoA dehydrogenase from the tapeworm E. granulosus. We have characterized the sequence, structure and location of EGR-03347 and investigated the immunoreactivity, immunogenicity and serodiagnostic potential of rEGR-03347. The results demonstrated rEGR-03347 as a potential vaccine against $E$. granulosus infection in dogs.

\section{Background}

Cystic echinococcosis (CE) is a zoonotic parasitic disease caused by the larvae of Echinococcus granulosus [1]. Dogs are the main definitive hosts of E. granulosus. In recent years, the numbers of pet dogs and abandoned dogs have increased yearonyear, leading to a large increase in the number of stray dogs, which has greatly increased the risk of transmission of CE [2]. Mammals such as sheep, cattle, camels, and donkeys are critical intermediate hosts in China [3]. The infection of intermediate hosts is 
caused by the ingestion of eggs containing oncospheres. Oncospheres can pass through the intestinal wall and enter the internal organs (liver and lung) via blood circulation to form cysts [4,5]. CE is a worldwide threat to human health, but also causes huge losses to animal husbandry $[6,7]$. The World Health Organization (WHO) has listed CE as one of the 17 neglected diseases in the 2020 strategic roadmap because of the prevalence and severity of the disease [8].

At present, patients are mainly cured by surgery; however, because of the long incubation period of echinococcosis, most cases of CE are diagnosed later, missing the optimal window for surgery [9]. Chemical drugs (praziquantel) are used mainly to deworm the definitive hosts (dogs) to block the transmission of the disease; however, it is time-consuming and difficult to implement at the grass-roots level, and there is also a risk of drug resistance. To date, the recombinant Eg95 vaccine against intermediate host (sheep) has been developed successfully. The protection rate of this vaccine in sheep is more than $90 \%$, and it has been used widely in key epidemic areas [10]. However, the Eg95 vaccine is only effective on the migratory stage, and has no protective effect on infected animals with formed cysts. The eggs produced by the mature gravid proglottids of $E$. granulosus in dogs are the key factors that infect people and animals. Therefore, the development of a dog anti-E. granulosus vaccine would satisfy the principle of effective prevention and control of the disease. Furthermore, the number of definitive host dogs in epidemic areas is always far less than that of intermediate host sheep; thus, the cost of definitive host vaccine prevention and control is also lower than that of the intermediate host. Therefore, the development of a canine anti-E. granulosus vaccine is a feasible prevention and control strategy, and will become the basis for echinococcosis prevention and control in the future $[11,12]$.

Cestodes lack a digestive system, and therefore must rely on their hosts to transport nutrients through the cortical syncytia [1]. Studies have shown that the biosynthetic ability of all parasitic flatworms (including tapeworms) is very limited, thus they must obtain most of the amino acids, nucleosides, and some lipids from the host body for reproduction and growth. Analysis of the genomic data of $E$. granulosus revealed that E. granulosus lacks the ability for de novo synthesis of lipids, purines, and most amino acids [13]; however, it has a complete set of tricarboxylic acid cycle enzymes, and the main oxidation function of this cycle is $\beta$ oxidation. 3-hydroxyacyl-CoA dehydrogenase is one of the main functional enzymes in the $\beta$ oxidation process. Previous work in our laboratory showed that the gene encoding 3-hydroxyacyl-CoA dehydrogenase was expressed in both protoscolex and adult $E$. granulosus, and its transcription level was higher in adult $E$. granulosus (data not shown). Therefore, it may be one of the key enzymes of fatty acid metabolism in E. granulosus, and could play an important role in the tapeworm's growth and development. To further reveal its biological characteristics and immune activity, in the present study, we aimed to clone and molecularly characterize the gene encoding 3-hydroxyacyl-CoA dehydrogenase of $E$. granulosus (termed EGR-03347), especially its tissue distribution. To evaluate the immune protective effect of recombinant (r)EGR-03347 on experimental dogs, we analyzed the changes in antibodies, and Th1 and Th2 cytokines. We also evaluated the worm reduction rate and segmental development inhibition rate in rEGR-03347-vaccinated dogs. This study provides a basis to reveal the function of EGR03347 and could lead to the successful development of a canine anti-E. granulosus vaccine. 


\section{Methods}

\section{Ethics Statement}

This study was reviewed and approved by the Care and Use of Laboratory Animals of the Xinjiang Academy of Agricultural and Reclamation Sciences (Shihezi, China) (XAARS; ApprovalNo. 2019-012, 9 April 2019). All animals were handled in strict accordance with the animalprotection laws of the People's Republic of China (a draft animal protection law was releasedon 18 September 2009) and the National Standards for Laboratory Animals in China (executed on 5 January 2002).

\section{Animals}

Six-to eight-week-old female specific pathogen free (SPF) mice were purchased from the Laboratory Animal Center of Xin jiang Medical University (Xingjiang, China). Nine 8-month old Beagle dogs of mixed breed were obtained from the Laboratory Animal Center of Sichuan Agricultural University.

\section{Parasites}

Hydatid cysts were collected from the livers of naturally infected sheep at an abattoir in Urumqi, Xinjiang Province, China. The fertility of the cysts was confirmed by observing PSCs within the cysts under light microscopy. Infertile cysts showed the absence of PSCs under either macroscopic or microscopic observation. Cyst walls from fertile/infertile cysts and PSCs were separated and treated as previously described [14]. Two thousand PSCs were cultured in $1 \mathrm{ml}$ of Roswell Park Memorial Institute (RPMI) 1640 medium with $10 \%$ bovine serum albumin (BSA; Hyclone, Logan, UT, USA), $100 \mathrm{U} / \mathrm{ml}$ penicillin, and 100 $\mu \mathrm{g} / \mathrm{ml}$ streptomycin (Sigma-Aldrich, St. Louis, MO, USA). Adult worms were obtained from a 8-month-old dog at 28 days after artificial infection with PSCs. BALB/c mice were prepared to produce polyclonal antibodies.

\section{Bioinformatics analysis}

The cDNA sequence of EGR-03347 (XP-024352997.1) was downloaded from NCBI, and the ProtParam tools on the ExPASY system (https://web.expasy.org/protparam/) were used to analyze the physicochemical parameters, signal peptide, and transmembrane regions of the putative encoded protein. Using the SOPMA online software forecasted the secondary structure(https://secondary-structureprediction.html), Tertiary (three-dimensional) structures were modeled through SWISS-MODEL (http://swissmodel.expasy.org/), and the IEDB online server was used to predict the B cell epitopes of EGR-03347. EGR-03347-similar sequences were aligned, and phylogenetic trees were constructed using the MEGA software (version 5.05) with the neighbor-joining ( $\mathrm{NJ}$ ) method. 


\section{expression, and purification of the non-transmembrane region of rEGR03347}

Total RNA was extracted from PSCs using an RNA-prep Pure Tissue Kit (Nanjing Vazyme Biotech, Nanjing, China), and a Reverse Transcription System Kit (Nanjing Vazyme Biotech) was used to synthesize first-strand cDNA. The full coding sequence of the non-membrane region of EGR-03347 was amplified from E. granulosus cDNA using primers F: (5'- CGGGATCCATGTCAGCCGGTGCTGG-3') and R: (5'- GACGTCGACTCACTGTTTTTCCTTGACAATGCGC-3'), which introduce BamHI and Sall restriction enzyme sites (underlined), respectively. The PCR protocol included 35 cycles of $94{ }^{\circ} \mathrm{C}$ for $30 \mathrm{~s}, 62{ }^{\circ} \mathrm{C}$ for $30 \mathrm{~s}$, and $72{ }^{\circ} \mathrm{C}$ for $30 \mathrm{~s}$. The PCR products were ligated into the pET32a (+) plasmid (Novagen, Darmstadt, Germany), and transformed into Escherichia coli BL21 (DE3) cells (Tiangen, Beijing, China), and induced with $1 \mathrm{mM}$ isopropyl-1-thio- $\beta$-D-galactopyranoside (IPTG) at $37^{\circ} \mathrm{C}$ for $6 \mathrm{~h}$. The inclusion bodies were resuspended in lysis buffer plus $8 \mathrm{M}$ urea and incubated on ice for $2.5 \mathrm{~h}$ to completely solubilize the protein. His-tagged rEGR03347 protein was purified using $\mathrm{Ni}^{2+}$ affinity chromatography with a Hisaffinity resin column (Bio-Rad, Hercules, CA, USA), and examined by $10 \%$ sodium dodecyl sulfatepolyacrylamide gel electrophoresis (SDS-PAGE). Protein concentrations were determined using a NanoDrop2000c spectrophotometer (Bio-Rad).

\section{Preparation of Polyclonal Antibodies}

Briefly, each mouse was inoculated subcutaneously with $50 \mu \mathrm{g} . \mathrm{mL}-1$ rEGR-03347 emulsified in Freund's complete adjuvant (FCA; Sigma), followed by three repeat inoculations every 2 weeks with $50 \mu \mathrm{g} \cdot \mathrm{mL}-1$ rEGR-03347 emulsified in Freund's incomplete adjuvant (Sigma), In the control group, mice were treated in the same way as immunized group except that the rEGR-03347 was replaced with (PBS). Sera were collected for antibody analysis at days 0 (pre-immunization), 14, 28, 42 and 56 days.

\section{Western blotting}

Total protein extracts of PSCs and rEGR-03347 were separated using $10 \%$ SDS-PAGE, and then transferred onto nitrocellulose membranes. The membranes were blocked using $5 \%(\mathrm{w} / \mathrm{v})$ skim milk at $37^{\circ} \mathrm{C}$ for $2 \mathrm{~h}$, and then incubated with E. granulosus sheep positive/ dog negative sera, anti rEGR-03347 mice sera, or pre-immunized mice sera $\left(1: 100 \mathrm{v} / \mathrm{v}\right.$ dilutions) overnight at $4{ }^{\circ} \mathrm{C}$. The following day, the membranes were washed and then incubated with horseradish peroxidase (HRP)conjugated sheep antimouse IgG or rabbit anti-dog IgG (1:5000 v/v dilution) for $2 \mathrm{~h}$. Signals were visualized using an ECL kit (Pierce ECL Western, Rockford, IL, USA) and Molecular Imaging system(BIO-RAD,USA) exposure.

\section{Immunolocalization}

To determine the tissue location of EGR-03347, fresh PSCs and adult worms were fixed in $4 \%$ paraformaldehyde phosphate buffer overnight, permeabilized using $1 \%$ Triton X-100 for 30 min, soaked in $0.01 \%$ Triton $\mathrm{X}-100$ for $1 \mathrm{~h}$ at $4{ }^{\circ} \mathrm{C}$, wash with $0.01 \times$ PBS three times, and then blocked using $5 \%$ $(\mathrm{w} / \mathrm{v})$ skim milk at $37^{\circ} \mathrm{C}$ for $2 \mathrm{~h}$. The sections were then incubated with anti-rEGR-03347 mouse lgG or native mouse lgG $\left(1: 100 \mathrm{v} / \mathrm{v}\right.$ dilutions in PBS + Tween 20 (PBST) overnight at $4{ }^{\circ} \mathrm{C}$, The sections were 
washed and incubated with fluorescein isothiocyanate (FITC)conjugated goat anti-mouse lgG $(\mathrm{H}+\mathrm{L})$ $(1: 1000 \mathrm{v} / \mathrm{v}$ dilution in PBST) at room temperature for $2 \mathrm{~h}$ in darkness. After four washes with PBST, the sections were examined under a fluorescence microscope (Leica, Wetzlar, Germany). Antibodies from preimmunized mice were used as a negative control.

\section{Quantitative real-time reverse transcription fluorescence- PCR analysis of EGR-03347 gene expression}

Total RNA and cDNA of PSCs and 28-day strobilated worms were obtained as described above. Quantitative real-time reverse transcription PCR was used to analyze expression profiles of EGR-03347 in PSCs and 28-day strobilated worms. The primers for EGR-03347 were 5'-TAGAGATGTGGGAGCGTTGC-3' and 5'-TCCGTAACCGCACTTTTTGC-3'. Expression of the actin gene was detected as an internal control for normalization. Primers specific to E. granulosus actin were 5'-CGCATCGGTCGTCTTGTGTT-3' and 5'CGGTAATCCTGTGGCTGTCAAT-3'. The data were analyzed using the 2- $\triangle \triangle$ CT method[15].

\section{Vaccination and parasite challenge}

We used nine Beagles dogs and placed them in three groups including Group 1 consisting of three dogs vaccinated with EGR-03347 mixed with Quil A; Group II consisting of three dogs vaccinated with the adjuvant Quil A (Sigma); and Group III comprised three dogs vaccinated with PBS as a control group. One dose of vaccine comprised $200 \mu \mathrm{g}$ of soluble recombinant EGR-03347 and $100 \mu \mathrm{g}$ of Quil A in $825 \mu \mathrm{L}$ of PBS. The mixture was stirred overnight at $4^{\circ} \mathrm{C}$ before vaccination. One week after the last vaccination boost, all dogs were orally challenged with 100000 PSCs. All the dogs in the above three trials were euthanized and necropsied 28 days after infection to collect and count $E$. granulosus worms, before the appearance of eggs, for safety reasons.

\section{Indirect ELISA}

The optimal concentrations of rEGR-03347 antigen and serum were assessed using a standard checkerboard titration procedure. The purified EGR-03347 protein was diluted to $5 \mu \mathrm{g} / \mathrm{ml}$ in $0.1 \mathrm{M}$ carbonate buffer $(\mathrm{pH} 9.6)$ and used as the antigen in the ELISA. The ELISA plates were coated with diluted antigen solution overnight at $4^{\circ} \mathrm{C}$. After washing with PBST, the plates were incubated with $5 \%$ skim milk for $2 \mathrm{~h}$ at $37^{\circ} \mathrm{C}$. The wells were washed thoroughly and incubated with $100 \mu \mathrm{L}$ of serum samples with two-fold dilutions (1:80) in PBST at $37^{\circ} \mathrm{C}$ for $1.5 \mathrm{~h}$. Following washing, 1:3000 dilutions of HRP-labeled rabbit anti-dog IgG (Solarbio, Beijing, China), were added to the plates, which were then incubated at $37^{\circ} \mathrm{C}$ for $1.5 \mathrm{~h}$. Then, the wells were washed again and incubated with the substrate $3,3^{\prime}, 5,5^{\prime}-$ Tetramethylbenzidine (TMB) (Tiangen, Beijing, China) at $37^{\circ} \mathrm{C}$ for $15 \mathrm{~min}$. Finally, color development was stopped with $100 \mu \mathrm{L}$ of $1 \mathrm{M} \mathrm{H} 2 \mathrm{SO} 4$, and the optical density at $450 \mathrm{~mm}$ (OD450) was determined.

\section{Detection of cytokines}

An ELISA Kit (Jianglaibio, Shanghai) was used to determine the five main cytokines in dogs (IL-1, IL-4, IL5, IL-6 and IFN- $y$ ). An ELISA strip in its aluminum foil bag was left at to equilibrate at room temperature for 60 minutes. The strip was removed from the bag and the standard sample well(dog sera). Each well 
received $50 \mu \mathrm{L}$ of dog sera at different concentrations respectively. Then, $100 \mu \mathrm{L}$ of HRP labeled antibody was added to each well. The reaction wells were sealed with a sealing film and incubated at $37^{\circ} \mathrm{C}$ for 60 min. The liquid was then discarded and the plate was patted dry using absorbent paper. Each well was filled with $350 \mu$ Lof PBST and left to strand for $1 \mathrm{~min}$. The detergent was removed, the plate was patted dry using absorbent paper, and the detergent washing step was repeated five times. Each well was then incubated with $50 \mu \mathrm{L}$ of substrate $A$ and $50 \mu \mathrm{L}$ of substrate $B$ at $37^{\circ} \mathrm{C}$ for $15 \mathrm{~min}$. Then, $50 \mu \mathrm{L}$ of termination solution was added into each well, and the OD value of each well at well was measured at $450 \mathrm{~mm}$ within $15 \mathrm{~min}$.

\section{Data analysis}

SPSS 22.0 software (IBM Corp, Armonk, NY, USA) was used for the statistical analyses, and GraphPad prism 6.0 (La Jolla, CA, USA) was used to visualize the data. At $p<0.05$, the results were considered statistically significant.

\section{Results}

\section{Molecular characteristics of EGR-03347}

The cDNA sequence of EGR-03347 contained 924 nucleotides and encoded a protein of 308 amino acids (aa) with no signal peptide, but with a single transmembrane region (aa 15-35). The protein had a predicted pl of 9.03 , a mass of $34 \mathrm{kDa}$, and an instability index of 34.22 . We identified 11 potential B cell linear epitopes in the amino acid sequence of EGR-03347(ep1: 4-16 aa; ep2: 51-55 aa; ep3: 69-88 aa; ep4: 90-92 aa; ep5: 107-109 aa; ep6: 134-142 aa; ep7: 144-145 aa; ep8: 171-174 aa; ep9: 219-223a a; ep10: 263-273 aa; and ep11: 281-304 aa). In the amino acid sequence of EGR-03347, the T cell epitopes were identified as being near amino acids $13-28,37-42,47-48,51-55,60-62,70-92,97-99$, 107-120, 125-126, 134-144, 154-155, 160-167, 182-198, 202-215, 229-237, 241-242, 249-258, and 262-274. Conserved domain analysis at the NCBI predicted that EGR-03347 contained a conserved domain and belonged to the FadB family. The secondary structure of EGR-03347 was predicted using the SOPMA online software. For the secondary structure, structural modeling predicted that $52.44 \%$ was ahelix, $26.06 \%$ was irregular coil, $15.64 \%$ was extended chain, and $5.86 \%$ was $\beta$-turn. There are five ahelices around four $\beta$-folds the structure of EGR-03347 (Fig. 1). Amino acid sequences of EGR-03347-like proteins from various species were retrieved from the GenBank database, and phylogenetic analysis showed that that EGR-03347 shares sequence identity with sequences from other species, but shows the highest sequence identity with the protein from Echinococcus multilocularis (Fig. 2).

\section{expression, and purification of the non-transmembrane region of EGR03347}

The cDNA encoding the non-transmembrane region of EGR-0347 was amplified from protoscoleces (PSCs). Recombinant (r)EGR-03347 was successfully expressed in E. coli. After purification, rEGR-03347, 
including the His-tag, showed a single band near the predicted size of $47 \mathrm{kDa}$ on $10 \%$ SDS-PAGE (Fig. 3).

\section{Western Blotting}

Western blotting showed that rEGR-03347 reacted with CE-positive dog sera and with anti-EGR-03347 mouse sera. respectively. Moreover, anti-EGR-03347 mouse sera IgGs recognized the native protein in total protein extracts of PSCs. As expected, no signal was observed when EGR-03347 was probed with sera from CE-negative dogs or pre-immunized mice (Fig. 4).

\section{Localization of EGR-03347 in different life cycles of E. granulosus}

The locations of native EGR-03347 in PSCs (Fig. 5A) and adult worms (Fig. 5B) were determined using immunofluorescence with specific polyclonal antibodies against rEGR-03347. EGR-03347 was located only in the tegument and invaginated sucker of the PSCs, and in the adult worms, EGR-03347 was distributed in the epidermis of the scolex, mature proglottids, and gravid proglottids. No specific fluorescence was observed in any sections when native mouse IgG was used.

\section{Differential expression of EGR-03347 mRNA in different developmental stages of worms}

qRT-PCR was used to detect the relative transcription of EGR-03347 mRNA. The results showed that EGR03347 mRNA was expressed in E. granulosus protoscolex and adult stage, with higher expression the adult stage $(p<0.05$; Fig. 6$)$.

\section{Indirect ELISA}

Building on the good antigenicity and immunoreactivity of rEGR-03347, the preliminary serodiagnostic potential of rEGR-03347 based on indirect ELISA was assessed. Sera from nine immunized dogs were measured at 14,28 , and 42 days after the first vaccination. Compared with the phosphate-buffered saline (PBS) control group, significant IgG responses were observed after the first immunization in the group immunized with rEGR-03347, and the peak was detected at 42 days postvaccination. One week after the last vaccination boost, moreover, after 28 days of protoscolex challenge, the antibody levels gradually increased and were significantly higher than those in the control group (Fig. 7).

\section{Canine cytokines induced by rEGR-03347}

Interleukin (IL)-1, IL-4, IL-5, IL-6, and interferon gamma (IFN- - ) levels were assessed using an ELISA kit at $0,14,28,42$ days and in the 28 days after challenge with protoscolex group, and other controls (Figure. 8). The serum IL-4 levels in the groups of vaccinated dogs group were significantly increased $(p<0.01)$ from two week post first and boost vaccination to week 6 (highest IL-4 level) compared with those in control dogs but not the 28 days after challenge with protoscolex group ( $p>0.05$; Fig. 8A). The serum IFN$Y$ levels in the vaccinated dogs increased gradually from initial vaccination until week $6(p<0.05)$, and then decreased during the challenge with protoscolex $(p>0.05$; Fig. 8B) The serum IL-6 levels in the vaccinated dogs increased gradually from initial vaccination until week $2(p<0.05)$, and then decreased 
$(p>0.05)$ during the next weeks (Fig. 8C). The serum IL-1 and IL-5 levels in the vaccinated dogs showed no significant changes (Fig. 8D and 8E).

\section{Vaccine efficacy of rEGR-03347 proteins combined with Quil A}

We vaccinated dogs with rEGR-03347 mixed with Quil A and used PBS as a control. We counted the number of worms in all groups of animals. Total and average numbers are presented in (Table 1). The results for was when emulsified with Quil A, the rEGR-03347 protein showed an $87.2 \%$ reduction in the number of cestodes in vaccinated dogs compared with PBS controls group, which induced an obvious level of protective efficacy in Beagles against $E$. granulosus infection at days 28 post-challenge in terms of inhibiting worm growth.

Table 1

Worm burden for dogs

\begin{tabular}{|llll|}
\hline Group (protein) & Dog No. & Number of worm & Reduction \% \\
\hline PBS & 1 & 2400 & \\
\hline & 2 & 3000 & - \\
\hline Average & 3 & 27000 & \\
\hline QuilA & & 14400 & - \\
\hline & 4 & 13000 & \\
\hline Average & 5 & 3200 & 87.2 \\
\hline EGR-03347 & 6 & 3800 & \\
\hline & 7 & 6666 & \\
\hline & 8 & 3600 & \\
\hline Average & 9 & 133 & \\
\hline Note: Reduction \% & & 1800 & \\
\hline average $\times 100 \%$
\end{tabular}

\section{The development of the worm}

We measured the sizes of 30 randomly chosen worms per experimental group and recorded the percentage of developed ( $£$ three segments) versus underdeveloped ( ${ }^{3}$ four segments) worms. The inhibition rate of the rEGR-03347 protein on the development and maturation of worms was $67.7 \%$ 
compared with PBS controls group. Thus, the rEGR-03347 vaccine induced an obvious protective efficacy in terms of inhibition of worm growth (Table 2).

Table 2. The development of worm segments in each experimental group

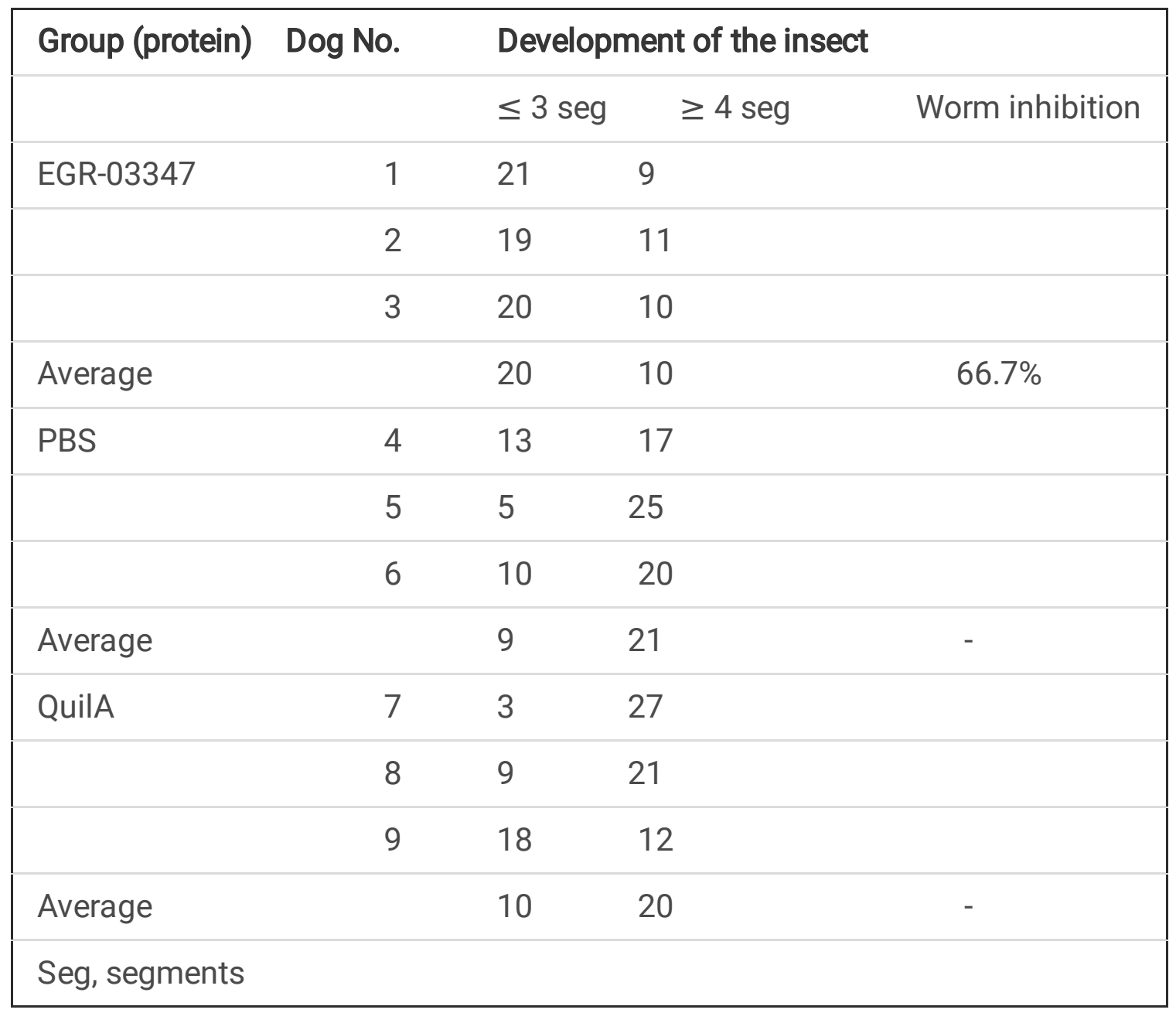

\section{Discussion}

Lipids are an important source of energy metabolism, including fatty acids, triglycerides, and cholesterol. The tricarboxylic acid cycle and $\beta$-oxidation process are the most important ways for most organisms to obtain energy. Fatty acid $\beta$-oxidation metabolism mainly involves acyl CoA and acetyl CoA. In the process of $\beta$-oxidation of fatty acids, 3-hydroxyacyl-CoA dehydrogenase (EGR-03347) is involved in the process of removing two carbon atoms from long-chain fatty acids, and is one of the key enzymes in fatty acid metabolism. This study used EGR-03347 to lay the foundation for the successful development of a canine anti-E. granulosus vaccine.

The development of vaccines against multicellular parasites, such as E. granulosus, is challenging compared with that for single celled organisms. However, the development of bioinformatics in recent decades has contributed to the improvement of the development of parasite vaccines, and 
immunoinformatic methods have promoted research into multi epitope vaccines. Multi epitope vaccines could induce a wide range of immune responses because of the presence of $B$ cell and $T$ cell epitopes. Therefore, the study of epitopes has played an important role in the construction of E. granulosus vaccines $[16,17]$. The results of the present study indicated that the amino acid sequence of EGR-03347 included 11 potential B cell linear epitopes, which suggested that EGR-03347 has great antigenic potential. The homology alignment of the EGR-03347 protein sequence showed that it was in the same branch with E. multilocularis, and its homology with other species was much lower. We observed that the EGR-03347 gene was expressed in the E. granulosus protoscolex and, especially, in adults. Moreover, immunofluorescence localization of EGR-03347 in the larvae and adults of E. granulosus showed that EGR-03347 was mainly distributed in the epidermis and sucker of PSCs, and in the scolex, mature, and gravid proglottis of the tapeworm, suggesting that EGR-03347 might play an important role in controlling and maintaining the specificity of the parasite life cycle [18]. Tapeworms like E. granulosus lack an intestinal tract, only possessing microvilli or microfibrils, which are the main sites for nutrient absorption and waste excretion [19]. Furthermore, the location of EGR-03347 in the suction cups and hooks of protocercus might be related to the energy demand attached to the host intestine, thus EGR-03347 might be involved in the interaction between the parasite and its host $[20,21]$. This suggested that EGR-03347 plays a key role in the growth and physiological activities of E. granulosus [22]. Tapeworms contain the complete metabolic pathways of fat, which could be used for the formation and development of the ascus and PSC $[23,24]$.

The western blotting results showed that the serum from mouse injected with rEGR03347 could recognize EGR-03347 among the natural proteins of PSCs, and the positive serum from dogs infected with $E$. granulosus could also recognize rEGR-03347, which indicated that rEGR-03347 had good immunoreactivity. In particular, the recognition of rEGR-03347 by the positive serum from dogs indicated that EGR03347 might be a component of excretory/secretory (ES) products of the E. granulosus[19]. However, the predicted amino acid sequence of EGR-03347 did not contain signal peptide; therefore, we speculated that EGR-03347 was an exocrine secretory protein, being released into extracellular secretion through exosomes [25].

In the present study, we used the mixed vaccine of EGR-03347 and Quil A to vaccinate the dogs. The Quil A adjuvant has been proven to be a key component in today's vaccines. Adding adjuvants to vaccine candidates could enhance the efficacy of the antigen, reduce the required dosage of the vaccine, make the host immune response more rapid, make $T$ helper cells bind more effectively to optimize the quality and persistence of the antibody response, or induce effector CD4 + or CD8 + T cells to kill pathogens in cells [26]. Therefore, this experiment used rEGR-03347 mixed with the Quil A adjuvant to induce a more effective immune response. After the third immunization, 100000 protoscoleces were taken orally in the PBS group as the control. The IgG titer of each immunization group was monitored. We observed that the antibody levels of the vaccine group increased gradually with prolonged immunization time, indicating that the specific antibodies were produced by stimulation with rEGR-03347. 
We noted that rEGR-03347 induced a Th1/Th2 cytokine response, and both Th1 and Th2 cytokines play an important role in the occurrence and development of echinococcosis. The Th2 response plays an important role in the emergence and growth of cysts, and plays a key role in the escape from 6immune surveillance [27], which is an important step in the development of a prophylactic E. granulosus vaccine. In the development of hydatid cysts, the Th1 immune response is dominant in the early stage of hydatid infection, and is related to protective immunity $[28,29]$. Th2 mainly produces IL-4, IL-5, and IL- 6 cytokines, while Th1 mainly produces IL1 and IFN-y. In echinococcosis, a large number of studies have shown that Th1 type and Th2 type cytokines co-exist. The Th2 response is conducive to the growth of the parasite, and the Th1 response inhibits the growth of the parasite and plays an immunoprotective role in the host $[30,31]$.

Li [32] found that by immunizing mice with rEg14-3-3, the secretion of IFN-y and IL-2 increased significantly, suggesting that Th1 might play a major role in the resistance to E. granulosus infection. Mohammad [33] immunized sheep and dogs with the rEGVac vaccine, and the IL-4 level in sheep and dogs increased significantly two weeks after immunization; however, the IL-4 level in serum decreased significantly after booster immunization, indicating that rEGVac has a significant inhibitory effect on the growth and development of adult PSCs.

In the present study, compared with the PBS control group, the secretion of IFNy and IL-4 in the serum of the enhanced immunized dogs increased significantly increased, which showed a downward trend after 28 days of protoscolex challenge. One of the characteristics of parasite infection is change the cytokine spectrum develop towards the Th2 type, such as producing IL-4, IL-5, and IL-6 [34]. In fact, the results may help to prevent host resistance to parasite resistance [35]. The increase in the total IgG level in dogs infected with protocercariae indicated that there may be a good humoral response. Eosinophils had shown the ability to clear protocercariae, and a large number of host reaction sites exist in some degenerated cysts, indicating that a Th2 type immune response might also play an important role in the anti-parasite response [36]. IFN- $y$ can activate macrophages to release NO, enhance the expression of $\mathrm{MHC}-I I$ molecules and the antigen presentation ability, thus inhibiting the protoscolex of E. granulosus. This supports the view that the rEGR-03347 vaccine plays an important role in anti $E$. granulosus infection.

According to a previous study [37], recombinant EgM9 and EgM123 were mixed with Quil A to vaccinate dogs. At 45 days after infection, fewer worms (17.1\%) developed 4 segments in the EgM9 vaccinated dogs compared with that in the control dogs, and EgM123 induced a significant protective efficacy in terms of worm burden reduction and suppression of worm growth and egg production at day 45 postinfection. No worms developed to the 4 segment stage and no worms produced eggs. In the present study, all dogs were sacrificed 28 days post challenge, before the appearance of eggs, for safety reasons. Compared with the PBS control group, the worm reduction rate was $87.19 \%$, It was better than the Salmonella vaccine EgA31-EgTrp(70\%-80\%)[38, but it was slightly lower than the recombinant vaccine EgM123(89.2\%)[37]. and the worm development to the mature stage was significantly decreased in rEGR03347 group, with an inhibition rate of $67.7 \%$. These results indicated that the rEGR-03347 vaccine could 
inhibit the growth and development of the tapeworms in canine intestines, which supported rEGR-03347 as a potential candidate vaccine for canine against $E$. granulosus infection.

\section{Conclusions}

In this study, we successfully designed a recombinant vaccine using an E. granulosus fatty acid metabolizing enzyme. We analyzed the structural characteristics and tissue distribution of EGR-03347, which provided novel insights into its biological function. We also performed an initial evaluation of the diagnostic potential of rEGR-03347 based on ELISA, indicating that it may be an effective antigen in $E$. granulosus infection. Th1 and Th2 cytokines were increased, and worm production development were inhibited in immunized dogs, which indicated that vaccination with rEGR-0347 played an important role in resisting $E$. granulosus infection. The implementation of the vaccine could effectively decrease echinococcosis in an epidemic area. Although the vaccine efficiency is high, we did not carry out an expanded scale field trial of the vaccine. The duration of the immunity provided by rEGR-03347 and its modified version will be evaluated in future studies.

\section{Declarations}

\section{Funding}

This research was funded by the National Natural Science Foundation of China(grant number 31860701) and the International Scientific and Technological Cooperation Projects of Xinjiang Production and Construction Corps (grant number 2021BC008, 2020BC007).

\section{Availability of data and materials}

The full-length DNA sequence of EGR-03347 has been deposited in GenBank database (https://www.ncbi.nlm.nih.gov/) under the accession number XP_024352997.1. Data supporting the conclusions of this article are included within the article. The data used and/or analyzed during the current study are available from the corresponding author upon reasonable request

\section{Ethics approval and consent to participate}

This study protocol was approved by the Research Ethics Committee and the

Animal Caring and Using Committee of State Key Laboratory of sheep Genetic Improvement and Healthy Production/Institute of Animal Husbandry and Veterinary

\section{Author Contributions}

Jinwen Xian, Ning Wang and Pengpeng Zhao participated in the design of the study, performed all experiments, collected and analyzed data, and completed manuscript preparation. Xu Ma helped in study design and implementation Yanyan Zhang, jimeng meng participated in collection of parasite specimens 
and carried out the immunofluorescence experiments, Zhengrong Wang and Xinwen Bo conceived of the study, participated in its design and coordination, and helped to interpret the results.

\section{Author details}

- State Key Laboratory of sheep Genetic Improvement and Healthy Production/Institute of Animal Husbandry and Veterinary, Xinjiang Academy of Agricultural and Reclamation Sciences, Shihezi 832000 , China.

- College of animal science and technology, Shihezi University, Shihezi 832000, China.

\section{Competing interests}

The authors declare that they have no competing interests

\section{References}

1. Brehm K, Koziol U. Echinococcus-Host Interactions at Cellular and Molecular Levels. Adv Parasitol. 2017;95:147-212.

2. Jenkins DJ, Macpherson CN. Transmission ecology of Echinococcus in wild-life in Australia and Africa. Parasitology. 2003;127 Suppl:S63-72.

3. Jenkins DJ. granulosus in Australia, widespread and doing well! Parasitol Int. 2006;55 Suppl:S203-6.

4. Eckert J, Deplazes P. Biological, epidemiological, and clinical aspects of echinococcosis, a zoonosis of increasing concern. Clin Microbiol Rev. 2004;17(1):107-35.

5. Carmena D, Cardona GA. Canine echinococcosis: global epidemiology and genotypic diversity. Acta Trop. 2013;128(3):441-60.

6. Budke CM, Deplazes Torgerson, P.R. Global socioeconomic impact of cystic echinococcosis. Emerg Infect Dis. 2006;12(2):296-303.

7. Cardona GA, Carmena D. A review of the global prevalence, molecular epidemiology and economics of cystic echinococcosis in production animals. Vet Parasitol. 2013;192(1-3):10-32.

8. Atkinson JA, Gray DJ, Clements AC, Barnes TS, McManus DP, Yang YR. Environmental changes impacting Echinococcus transmission: research to support predictive surveillance and control. Glob Chang Biol. 2013;19(3):677-88.

9. Brunetti E, Kern P, Vuitton DA. Writing Panel for the WHO-IWGE. Expert consensus for the diagnosis and treatment of cystic and alveolar echinococcosis in humans. Acta Trop. 2010;114(1):1-16.

10. Gauci C, Heath D, Chow C, Lightowlers MW. Hydatid disease: vaccinology and development of the EG95 recombinant vaccine. Expert Rev Vaccines. 2005;4(1):103-12.

11. Craig PS, Larrieu Control of cystic echinococcosis/hydatidosis: 1863-2002. Adv Parasitol. 2006;61:443-508. 
12. Zhang W, McManus DP. Vaccination of dogs against granulosus: a means to control hydatid disease? Trends Parasitol. 2008;24: 419-24.

13. Stantcheva N, Riddiford N, Rosenzvit M, Salinas G, Wasmuth JD, Zamanian M. The genomes of four tapeworm species reveal adaptations to parasitism. Nature. 2013;496(7443):57-63.

14. Zhan J, Song H, Wang N, Guo C, Shen N, Hua R, etal. Molecular and functional characterization of inhibitor of apoptosis proteins (IAP, BIRP) in Echinococcus granulosus. Front Microbiol. 2020;11:729

15. Livak KJ, Schmittgen TD. Analysis of relative gene expression data using real-time quantitative PCR and the $2^{-\triangle \Delta C T}$ Methods. 2001;25:402-8

16. Stephenson R, You H, McManus DP, Toth I. Schistosome Vaccine Adjuvants in Preclinical and Clinical Research. Vaccines (Basel). 2014;2(3):654-85.

17. Sanches RCO, Tiwari S, Ferreira LCG, Oliveira FM, Lopes MD, Passos MJF, etal. Immunoinformatics Design of Multi-Epitope Peptide-Based Vaccine Against Schistosoma mansoni Using Transmembrane Proteins as a Target. Front Immunol. 2021;12:621706.

18. Huang $\mathrm{Y}$, Liu J, Kang $\mathrm{H}$, Chen J, Wang $\mathrm{L}$, Chen $\mathrm{A}$, The genome of the hydatid tapeworm E. granulosus. Nat Genet. 2013;45:1168-75.

19. Xin Q, Yuan M, Lv W, Li H, Song X, Lu J, etal.; Molecular characterization and serodiagnostic potential of Echinococcus granulosus Parasit Vectors. 2021;14(1):105.

20. Coltorii E.A, Varela-Díaz VM. granulosus: penetration of macromolecules and their localization on the parasite membranes of cysts. Exp Parasitol. 1974;35:225-31.

21. Deng C, Sun J, Li X, Wang L, Hu X, Wang X, etal. Molecular identifification and characterization of leucine aminopeptidase 2, an excretory-secretory product of Clonorchis sinensis. Biol. Rep. 2012, 39, 9817-9826.

22. Wang N, Zhu H, Zhan J, Guo C, Shen N, Gu X, etal. Cloning, expression, characterization, and immunological properties of citrate synthase from granulosus. Parasitol Res. 2019;118(6):18111820.

23. Song SM, Park JH, Kim J, Kim SI, Hong YC, Kong HH, etal. Identifification and characterization of Paragonimus westermani leucine aminopeptidase. Int. 2008, 57, 334-341.

24. Irshadullah M, Rani M. Comparative studies on the biochemical composition and polypeptide profiles of the cyst walls from sterile and fertile hydatid cysts of granulosus from buffalo host. Helminthologia. 2011;48:88-93.

25. Siles-Lucas M, Sánchez-Ovejero C, González-Sánchez M, González E, Falcón-Pérez JM, Boufana B. Isolation and characterization of exosomes derived from fertile sheep hydatid cysts. Vet Parasitol. 2017;236:22-33.

26. Reed SG, Orr MT, Fox CB. Key roles of adjuvants in modern vaccines. Nat Med. 2013;19(12):1597608.

27. McManus DP, Gray DJ, Zhang W, Yang Y. Diagnosis, treatment, and management of echinococcosis. BMJ. 2012;344:e3866. 
28. Reiner SL. Parasites and T helper cell development: Some insights. Parasitol Today. 1994; 10: 485-8.

29. Amri M, Mezioug D, Touil-Boukoffa C. Involvement of IL-10 and IL-4 in evasion strategies of granulosus to host immune response. Eur Cytokine Netw. 2009;20(2):63-8.

30. Riganò R, Profumo E, Buttari B, Teggi A, Siracusano A. Cytokine gene expression in peripheral blood mononuclear cells (PBMC) from patients with pharmacologically treated cystic echinococcosis. Clin Exp Immunol. 1999;118(1):95-101.

31. Riganò R, Profumo E, Di FeliceG, Ortona E, Teggi A, Siracusano A. In vitro production of cytokines by peripheral blood mononuclear cells from hydatid patients. Clin Exp Immunol. 1995;99(3):433-9.

32. Li ZJ, Wang YN, Wang Q, Zhao W. granulosus 14-3-3 protein: a potential vaccine candidate against challenge with E. granulosus in mice. Biomed Environ Sci. 2012;25(3):352-8.

33. Pourseif MM, Moghaddam G, Nematollahi A, Khordadmehr M, Naghili B, Dehghani J, etal. Vaccination with rEGVac elicits immunoprotection against different stages of granulosus life cycle: A pilot study. Acta Trop. 2021;218:105883.

34. Baz A, Ettlin GM, Dmatteis S. Complexity and function of cytokine responses in experimental infection by granulosus. Immunobiology 2006;211:3-9.

35. Gottstein B, Soboslay P, Ortona E, Wang J, Siracusano A, Vuitton DA. Immunology of Alveolar and Cystic Echinococcosis (AE and CE). Adv Parasitol. 2017;96:1-54.

36. Rostami-Rad S, Jafari R, Yousofi Darani H. Th1/Th2-type cytokine profile in C57 black mice inoculated with live granulosus protoscolices. J Infect Public Health. 2018;11(6):834-839.

37. Zhang ZZ, Guo G, Li J, Shi BX, Zhao L, Guo BP, etal. Dog vaccination with EgM proteins against granulosus. Infect Dis Poverty. 2018;7(1):61.

38. Petavy AF, Hormaeche $\mathrm{C}$, Lahmar S, Ouhelli $\mathrm{H}$, Chabalgoity A, Marchal $\mathrm{T}$, An oral recombinant vaccine in dogs against Echinococcus granulosus, the causative agent of human hydatid disease: a pilot study. PLoS Negl Trop Dis. 2008 Jan 16;2(1):e125.

\section{Figures}


$\begin{array}{rrrrrrr}10 & 20 & 30 & 40 & 50 & 60 & 70 \\ \mid & \mid & \mid & \mid & \mid & \mid & \mid\end{array}$

MLRSKVSCGNGNLTRFVSWNAVIGCGLIGSGIAAVSAGAGNMVVLSSSOORLKQQSKGRVSGVLCKLAKN eeeeeccccccchhhheeeeeeeettcccchhhhhhhhttceeeeeecchhhhhhhhhhhhhhhhhhhht KSGGFADSANARIKFTTDITTAVSOSQLLWEAVTEDLQIKKSLLFOSVERLAPNSCVLASITSALSWEIA

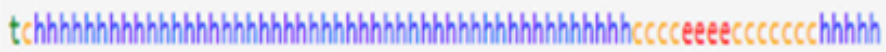
EVLSRKESFGGLLFFIPVRVKKLVEITRCRHTSEDTLKTLVAFAESLGKTVIHCKDTPGFIVIRLLVPYL

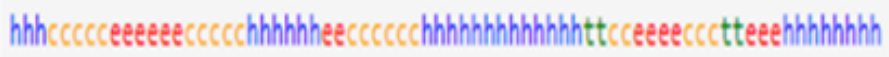
LEAIENERCDASLDDIETAMRLGGGPMGPFELADHVGLTLLLHILVHWERHPEERAFRLIATVRKFV

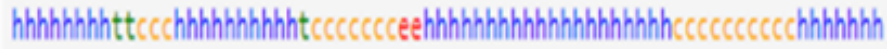
AHGRFGKKCGYGFFKYDQSGRIVKEXQ

httcccecccceeeecttcceeeect

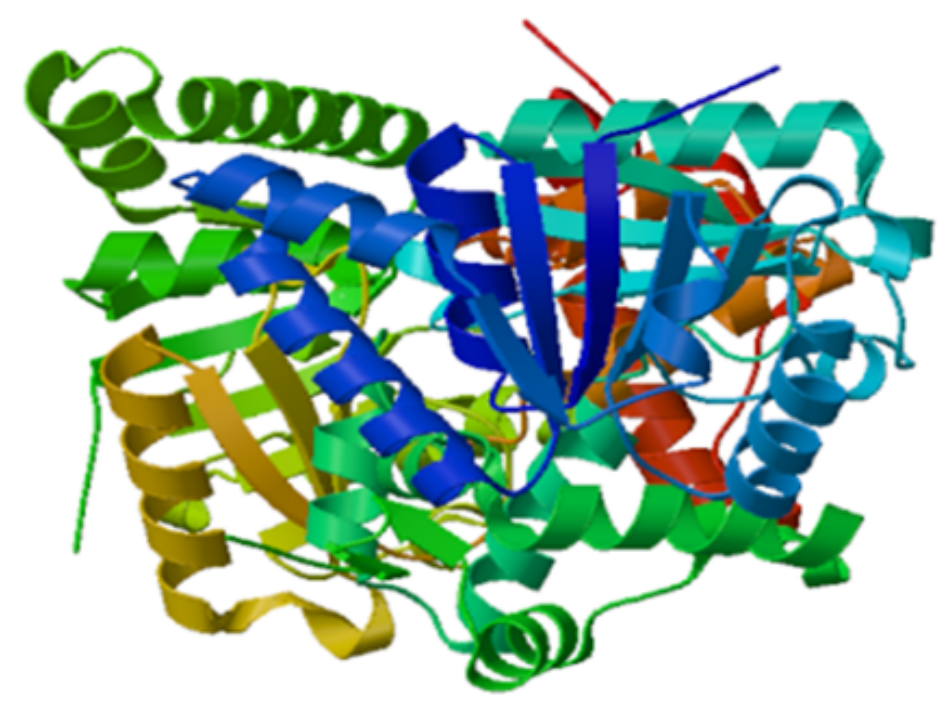

\section{Figure 1}

Prediction of the secondary and three-dimensional structure of the EGR-03347 protein.

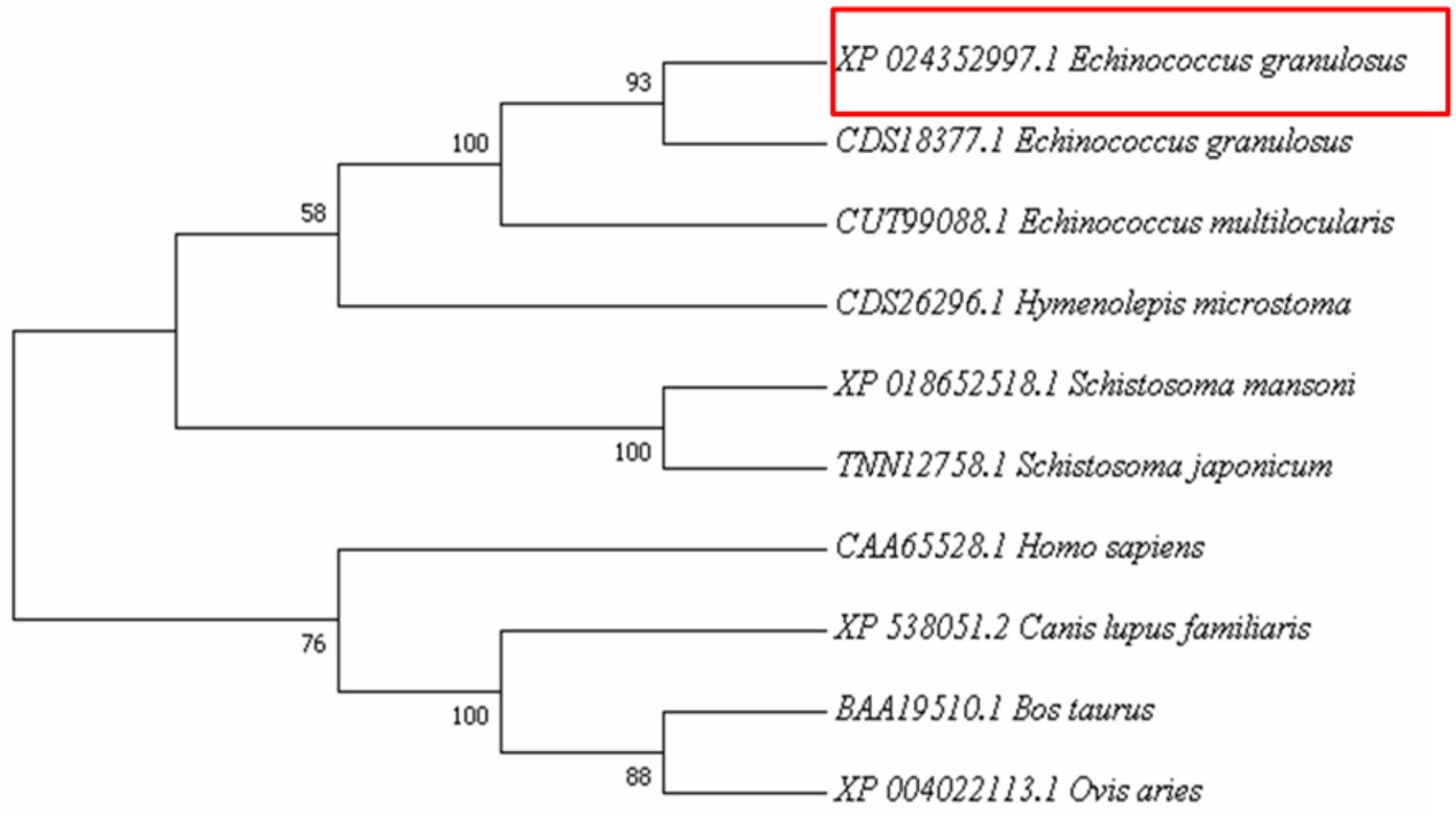

\section{Figure 2}

Phylogenetic tree of EGR-03347 and other species, the evolutionary tree was constructed by Neighbor Joining method in the MEGA software. The following sequences were retrieved from the GenBank databases:Echinococcus granulosus (GenBank:XP_024352997.1); Echinococcus granulosus(GenBank:CDS18377.1); Echinococcus multilocularis(GenBank:CUT99088.1); Taenia asiatica(GenBank:CUT99088.1); Hymenolepis microstoma(GenBank:CDS26296.1); Schistosoma 
mansoni(GenBank:XP_018652518.1); Schistosoma japonicum(GenBank:TNN12758.1 ); Homo sapiens(GenBank:CAA65528.1);Bos taurus(GenBank:BAA19510.1 ); Ovis aries

(GenBank:XP_004022113.1); Canis lupus familiaris(GenBank:XP_538051.2)

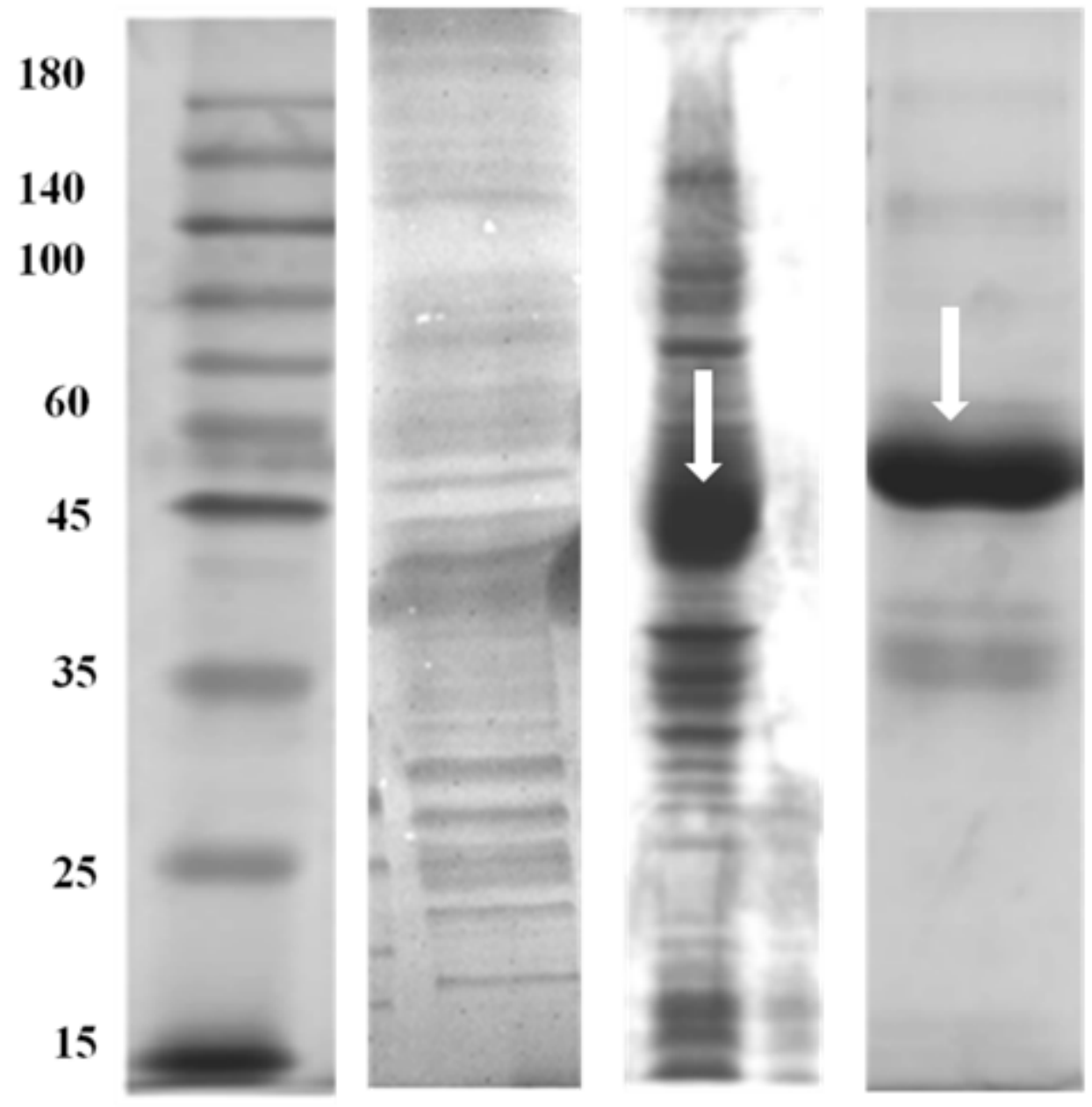

\section{Figure 3}

expression and purification of rEGR-03347 protein; Lane M, protein marker(KDa); Lane 1, total proteins from Escherichia coli BL21 (DE3) transformants harbouring pET32a(+)-EGR-03347; Lane 2, total proteins from Escherichia coli BL21 (DE3) transformants harbouring pET32a(+)-EGR-03347 induced by isopropyl$\beta$-D-1-thiogalactopyranoside (IPTG); Lane 3, sample after Ni2+ column purifification of recombinant Histagged protein 


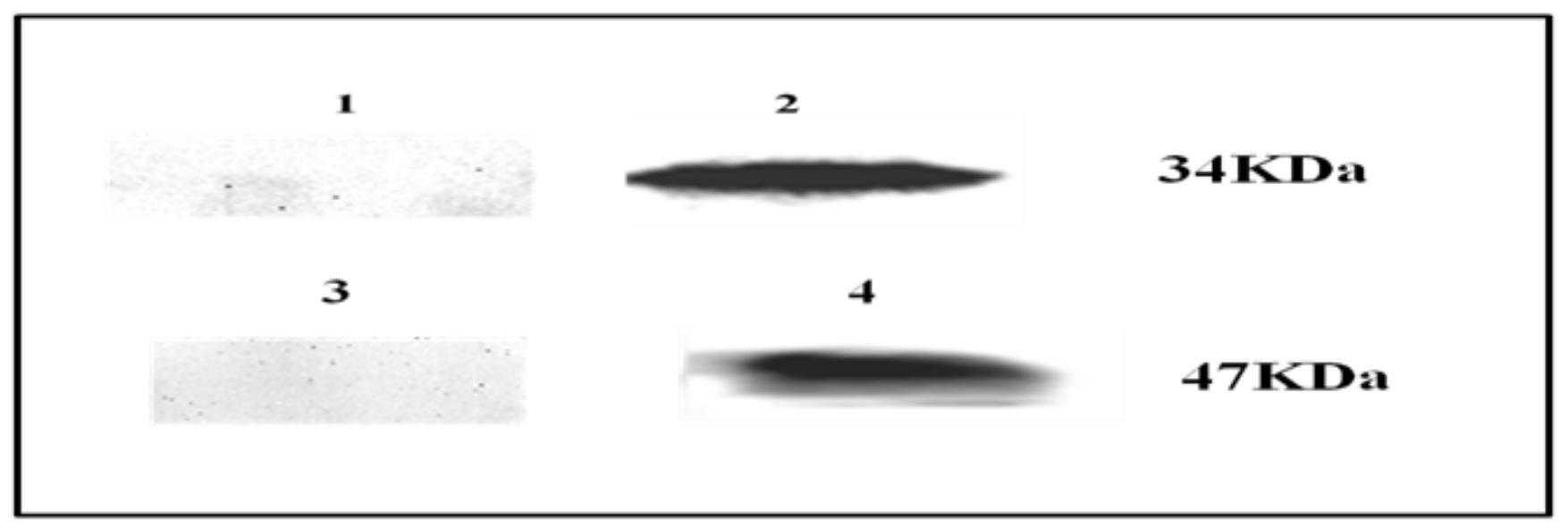

Figure 4

western blot analysis. Lane 1, total protein extracts of PSCs probed with pre-immunised mice sera; Lane 2, total protein extracts of PSCs probed with anti-rEGR-03347 mice sera; lane 3, purified rEGR-03347 probed with non-infected dog sera; Lane 4, purified rEGR-03347 probed with sera from cystic echinococcosis (CE)-positive dog sera 


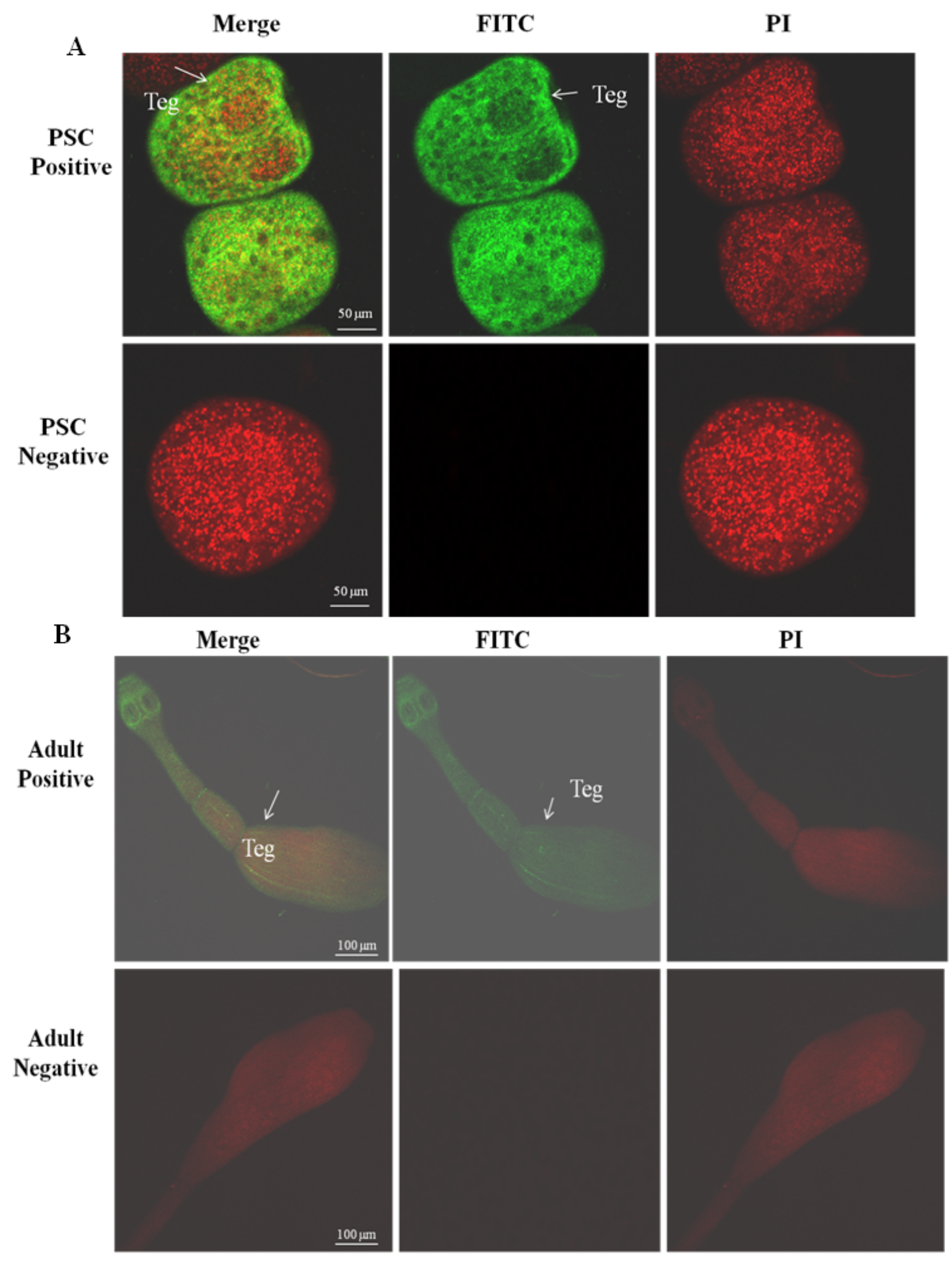

Figure 5

immunolocalization of EGR-03347 in different stages of E. granulosus; EGR-03347 in the protoscolex, and adult worm labeled by incubation with specifific anti-rEGR-03347 IgG (positive), or preimmune serum (negative), then with FITC-conjugated anti-micet lgG. (A)Scale bars: $50 \mu \mathrm{m}$ (B)Scale bars: $100 \mu \mathrm{m}$. Teg Tegumen 


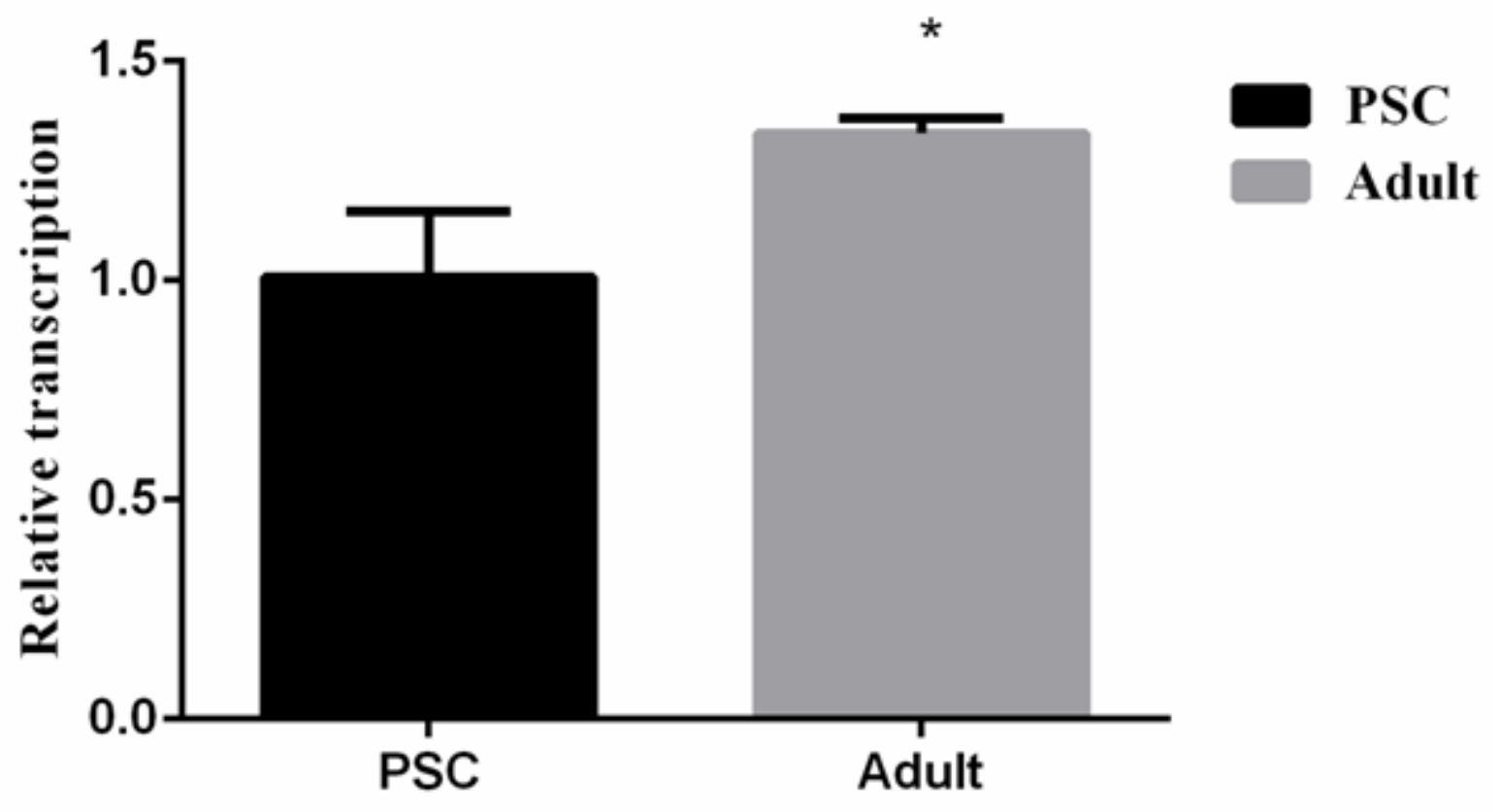

Different developmental stages of E.g

Figure 6

expression analysis of EGR-0347 mRNA in different developmental stages

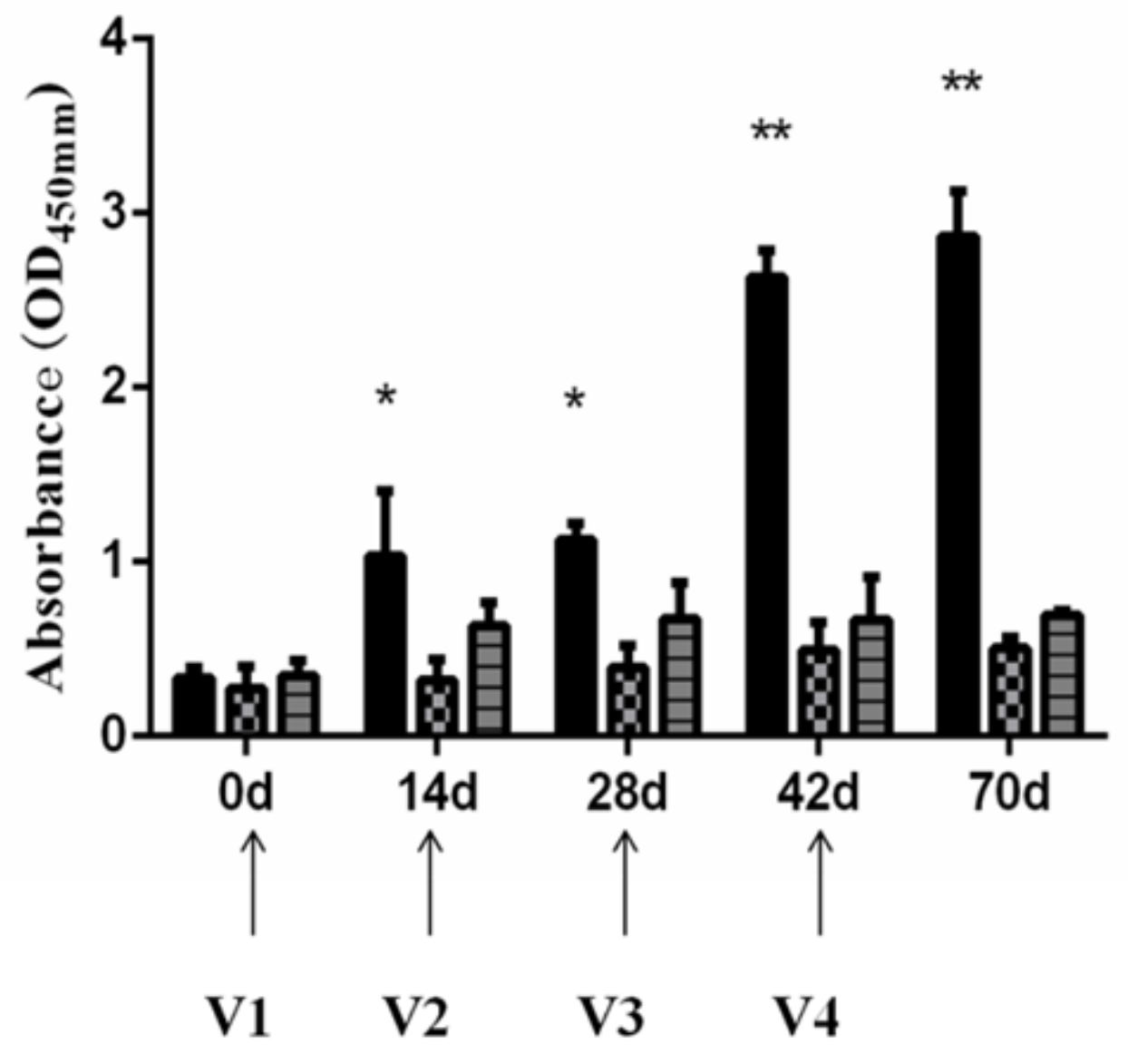

EGR-03347

D PBS

$\boxminus$ QuilA 
Figure 7

changes of antibody IgG level in dogs

A
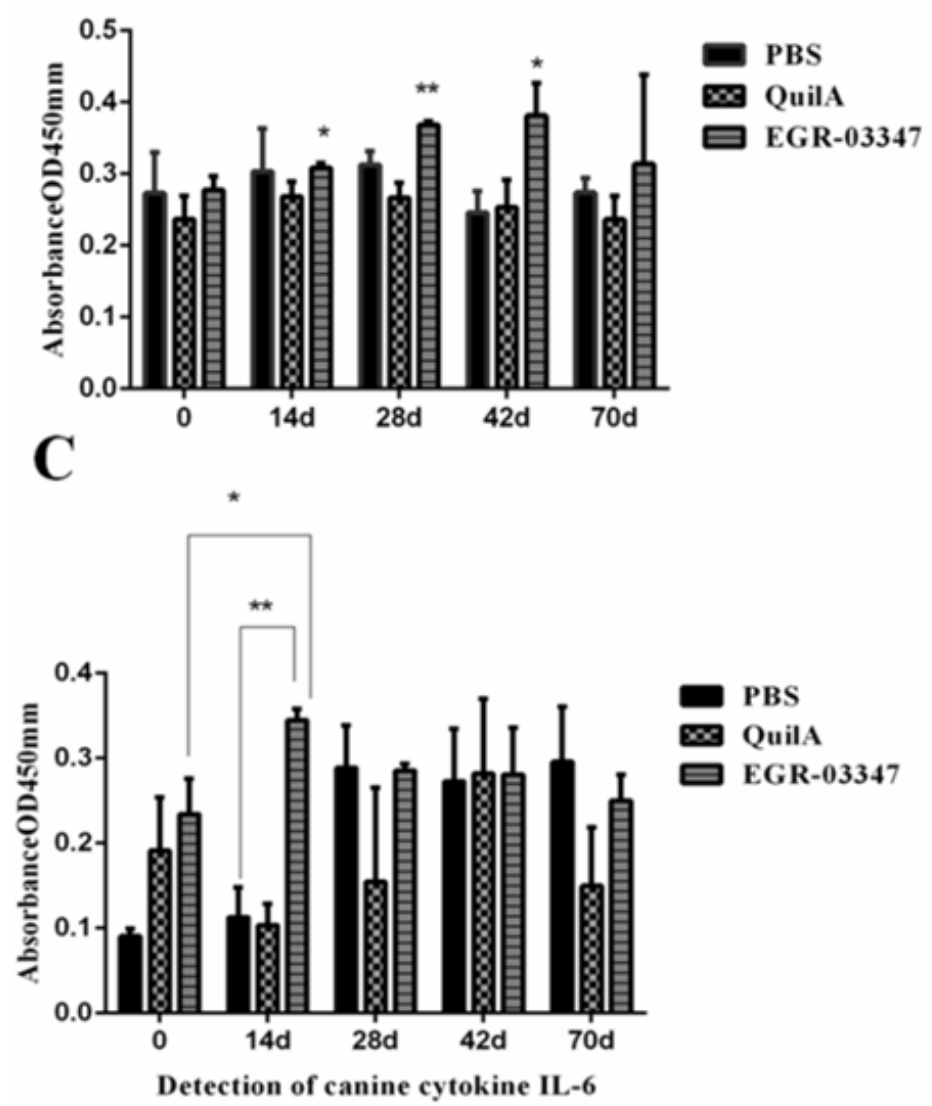

E
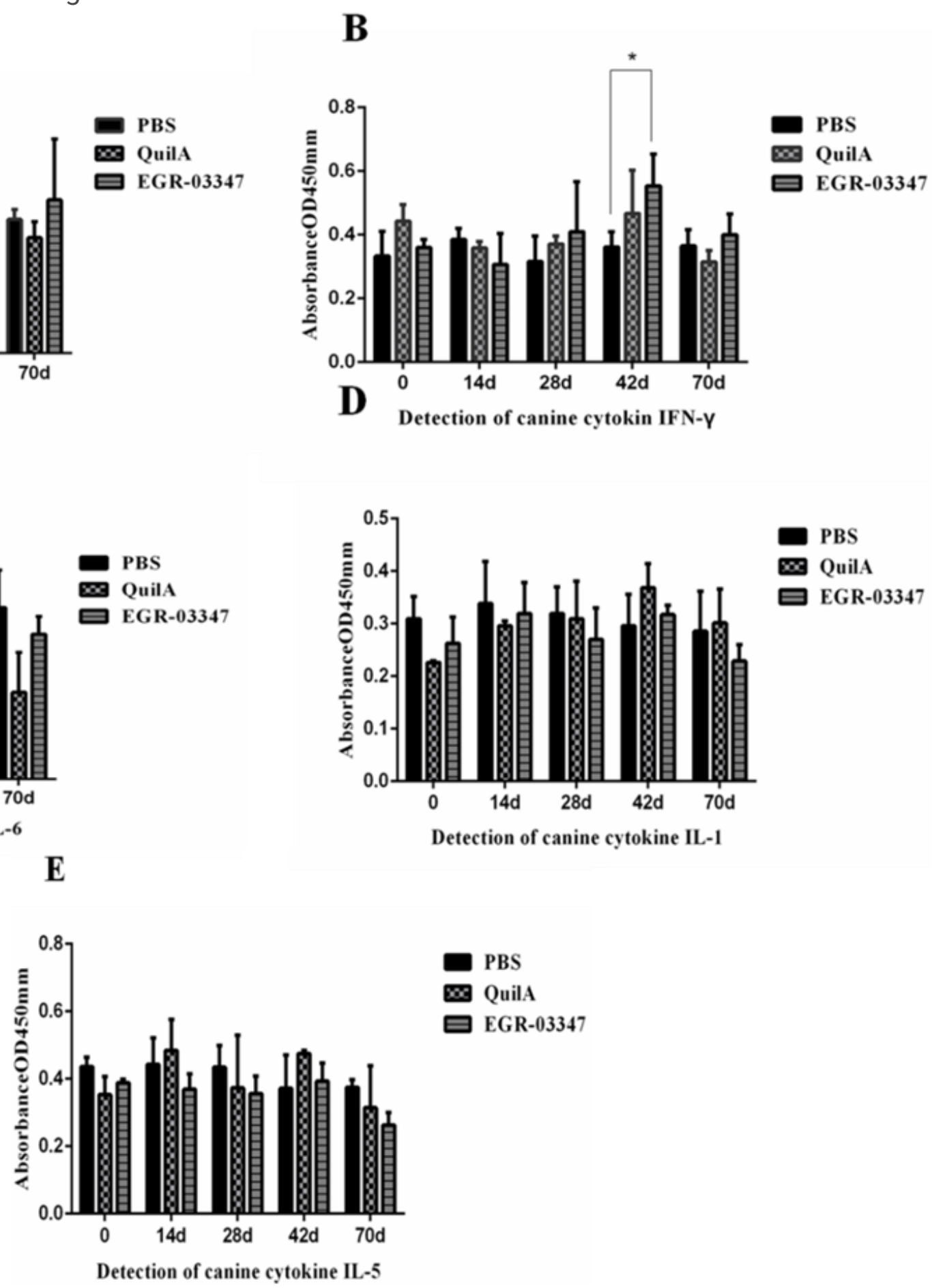

Figure 8

the results of ELISA for different cytokines in dog

Supplementary Files 
This is a list of supplementary files associated with this preprint. Click to download.

- renamedbe15b.docx 